\title{
Is K KP a luminous condition?
}

\section{Martin Smith ${ }^{1}$}

Received: 6 October 2021 / Accepted: 13 January 2022 / Published online: 16 February 2022

() The Author(s) 2022

\section{Abstract}

One of the most intriguing claims in Sven Rosenkranz's Justification as Ignorance is that Timothy Williamson's celebrated anti-luminosity argument can be resisted when it comes to the condition $\sim \mathrm{K} \sim \mathrm{KP}$ - the condition that one is in no position to know that one is in no position to know $\mathrm{P}$ (for some proposition $\mathrm{P}$ ). In this paper, I critically assess this claim.

\section{Introduction}

The idea at the heart of Sven Rosenkranz's Justification as Ignorance is that one has justification for a proposition $\mathrm{P}$ iff one is in no position to know that one is in no position to know P. If we let J abbreviate 'One has justification for...' and $\mathrm{K}$ abbreviate 'One is in a position to know...', we can put the idea like this: JP $<->\sim \mathrm{K} \sim \mathrm{KP}$. Rosenkranz claims a number of benefits for this view-and one of the most striking is that it allows us to defeat Timothy Williamson's well-known anti-luminosity argument and maintain that justification is a luminous condition. A condition is said to be luminous iff, whenever it obtains, one is in a position to know that it does. Justification will be a luminous condition iff we have the theorem JP $->\mathrm{KJP}$ which, for Rosenkranz, corresponds to the theorem $\sim \mathrm{K} \sim \mathrm{KP}->\mathrm{K} \sim \mathrm{K} \sim \mathrm{KP}$.

Williamson's argument-outlined in Chap. 4 of Knowledge and Its Limits (2000) - purports to show that there are no non-trivial luminous conditions. According to Williamson, for any condition that obtains in some cases and not others, there will be a possible case in which it obtains but one is in no position to know that it does. A number of conditions have been supposed, by philosophers, to be

Martin Smith

Martin.Smith@ed.ac.uk

1 University of Edinburgh, Edinburgh, UK 
luminous - phenomenal states, seemings, moral obligations, meanings and, indeed, justification, with epistemic 'internalists' claiming that we have special, privileged access to our own justificatory status. ${ }^{1}$ If Williamson's argument is successful-and many think that it is - then a lot of traditional philosophical theorising turns out to be misguided. And if Rosenkranz has genuinely hit upon a way of resisting the argument, at least when it comes to justification, then this too would represent a very significant result.

I am inclined to think that the anti-luminosity argument, as standardly formulated, really does falter when it comes to $\sim \mathrm{K} \sim \mathrm{KP}$. As I shall show, however, there is a fix available - the argument can be adapted to get around this problem. My primary aim here is to set out a new variant of the anti-luminosity argument, relying on slightly different assumptions to the original, which will work as effectively against $\sim \mathrm{K} \sim \mathrm{KP}$ as any other candidate non-trivial luminous condition. This new variant of the argument may be of some independent interest and may be better able to withstand certain extant objections to the original-but I won't explore this here.

What is it to be in a position to know a proposition? Some take this to imply that one could come to know the proposition without needing to conduct further empirical inquiry - come to know the proposition purely by reflection, introspection, a priori reasoning, etc. No detailed account of being in a position to know will be offered here, but I assume at a minimum that if one is in a position to know $\mathrm{P}$, and one has done everything that one is in a position to do to determine whether $\mathrm{P}$, then one knows P (Williamson, 2000, pp95, Rosenkranz, 2021, pp39). Call this $\mathrm{PK}_{1}$. This already gives us the result that being in a position to know is factive $-\mathrm{KP}->\mathrm{P}$.

I will table two further related assumptions, both of which are appealed to by Rosenkranz: First, if one has done everything that one is in a position to do to determine whether $\mathrm{P}$, then one has also done everything that one is in a position to do to determine whether $\sim$ P. Second, if one has done everything that one is in a position to do to determine whether one is in a position to know $\mathrm{P}$, then one has also done everything that one is in a position to do to determine whether P. In other words, doing everything that one is in a position to do to determine whether KP involves doing everything that one is in a position to do to determine whether P (plus, potentially, more). The former assumption, which we can call $\mathrm{PK}_{2}$, might be regarded as trivial. The latter, $\mathrm{PK}_{3}$, is not a triviality but is I think defensible. ${ }^{2}$ In any case, I won't further discuss these assumptions here (see, Rosenkranz, 2021, Sect. 3.2, where these assumptions correspond, respectively, to his $2 \mathrm{a}$ and $2 \mathrm{~b}$ ).

\footnotetext{
1 In the taxonomy of Pryor (2001, Sect. 3), this claim is characteristic of access internalism, while simple internalists claim only that one's justificatory status supervenes upon facts to which one has special, privileged access (a claim that would still presumably run afoul of the anti-luminosity argument).

2 Suppose I'm sitting indoors, with the curtains drawn, and considering the proposition that there is currently a yellow car parked outside. Can't I easily tell that I'm in no position to know this, without doing everything I' $m$ in a position to do to determine whether it's true-without, for instance, drawing back the curtains and looking out the window? What this sort of example highlights is that if 'being in a position to know' is interpreted purely in terms of reflective inquiry, then 'doing everything that one is in a position to do' must be interpreted in the same way. In this example, I can easily tell that I'm in no position to know by reflection that there is a yellow car outside-but there is nothing I can do by reflection to determine whether there is a yellow car outside, in which case the consequent of $\mathrm{PK}_{3}$ is trivially satisfied.
} 


\section{The anti-luminosity argument}

There is no single, canonical way of presenting the anti-luminosity argument, with recent presentations departing from Williamson's original in various respects. The version I will focus on is close to the one used by Rosenkranz (Sect. 4.3), though elaborated in the manner suggested by Srinivasan (2013). Let $C$ be a candidate nontrivial luminous condition. Imagine a sequence of cases $\alpha_{1}, \alpha_{2} \ldots$ linking a case in which $C$ clearly obtains to a case in which $C$ clearly obtains and which proceeds, through time, tracking minute, incremental changes to a relevant dimension, in a such a way that adjacent cases can be made arbitrarily similar to one another. ${ }^{3}$ The first assumption to be noted (call it $\mathrm{AL}_{1}$ ) is that it is, in fact, possible to construct a sequence of this kind for condition $C$. Suppose that we do have such a sequence and suppose that, throughout the sequence, I am using the optimal available method to detect condition $C$, and am doing everything that $\mathrm{I}$ am in a position to do to determine whether it obtains. As a result, if $\mathrm{I}$ am in a position to know that $C$ obtains in any given case in the sequence, then, by $\mathrm{PK}_{1}$, I $d o$ know that $C$ obtains in that case.

The second assumption that drives the argument (call it $\mathrm{AL}_{2}$ ) is that if $\mathrm{I}$ believe that $C$ obtains in a case $\alpha_{x}$, then I could easily believe that $C$ obtains in the adjacent cases $\alpha_{x-1}$ and $\alpha_{x+1}$. AL $\mathrm{AL}_{2}$ is supposed to be motivated by the fact that our powers of discrimination are limited and, since adjacent cases can be made arbitrarily similar, there is some point at which I would simply have no reliable way of telling them apart. The third assumption of the argument $\left(\mathrm{AL}_{3}\right)$ is that if $\mathrm{I}$ know that $C$ obtains in a case $\alpha_{x}$, then I could not easily have falsely believed that $C$ obtains in the next case $\alpha_{x+1} . \mathrm{AL}_{3}$ is supposed to be motivated by a kind of safety or margin-for-error requirement on knowledge - in order for one to know a proposition $\mathrm{P}$, it is necessary that one could not easily have believed P falsely, by the same method, in similar cases.

We now have the tools needed to prove that $C$ cannot be a luminous condition. By classical logic, either $C$ or $\sim C$ will obtain in each case in the sequence. As a result, there must be a last case in the sequence - call it $\alpha_{n}$-in which $C$ obtains and which is adjacent to the first case $-\alpha_{n+1}$-in which $\sim C$ obtains. If I don't believe that $C$ obtains in $\alpha_{n}$, then I don't know it. But if I do believe that $C$ obtains in $\alpha_{n}$, then, by $\mathrm{AL}_{2}$, I could easily believe that $C$ obtains in $\alpha_{n+1}$ from which it follows, by $\mathrm{AL}_{3}$, that my belief in $\alpha_{n}$ does not qualify as knowledge. Either way, $\alpha_{n}$ is a case in which $C$ obtains, even though I fail to know that it does.

Even if I believe that $C$ obtains right up to $\alpha_{n}$ and then cease to believe it in $\alpha_{n+1}$ - that is, even if my belief switches at exactly the point that $C$ does-this would just be good fortune. Given the limits on my powers of discrimination, I could easily have continued to believe that $C$ obtains in $\alpha_{n+1}$ even if, in actual fact, I don't. Talk about what could 'easily happen' can be usefully understood as existentially quantifying over possibilities that are very similar to actuality. For any case in the

\footnotetext{
${ }^{3}$ Cases are considered to be 'centred' possible worlds-triples consisting of a world, a subject and a time (Williamson, 2000, pp94). The cases in the sequence are distinguished only by the time variable, which changes in arbitrarily small increments while the world and subject are held constant.
} 
sequence $\alpha_{x}$, say that a possible case $\beta_{x}$ is a near duplicate of $\alpha_{x}$ iff $\alpha_{x}$ and $\beta_{x}$ are (i) identical with respect to whether $C$ obtains, with respect to the underlying dimension that drives the sequence and with respect to the fact that I'm doing everything I am in a position to do to determine whether $C$ obtains and (ii) are very similar in all other respects. Note that, in any near duplicate of a case in the sequence, just as in the cases themselves, I will know that $C$ obtains whenever I' $m$ in a position to know that it does. ${ }^{4}$ We can now translate talk about easy possibility into talk about near duplicates: what it means to say that I could easily believe that $C$ obtains in a case $\alpha_{x}$ is that there is a near duplicate of $\alpha_{x}$ (which could be $\alpha_{x}$ itself) in which I $d o$ believe that $C$ obtains.

Given this translation, $\mathrm{AL}_{2}$ could be written as follows: if I believe that $C$ obtains in a case $\alpha_{x}$ then there is a near duplicate $\beta_{x+1}$ of $\alpha_{x+1}$ and a near duplicate $\beta_{x-1}$ of $\alpha_{x-1}$ such that I believe that $C$ obtains in $\beta_{\mathrm{x}+1}$ and $\beta_{x-1}$. And $\mathrm{AL}_{3}$ becomes: if I know that $C$ obtains in a case $\alpha_{x}$, then I don't falsely believe that $C$ obtains in any near duplicate of $\alpha_{x+1}$. We can now retrace the argument. If I don't believe that $C$ obtains in $\alpha_{n}$, then I don't know it. If I do believe that $C$ obtains in $\alpha_{n}$, then, by $\mathrm{AL}_{2}$, there is near duplicate $\beta_{n+1}$ of $\alpha_{n+1}$ in which I believe that $C$ obtains. By the definition of a near duplicate, $\sim C$ obtains in $\beta_{n+1}$ from which it follows, by $\mathrm{AL}_{3}$, that my belief that $C$ obtains in $\alpha_{n}$ does not qualify as knowledge. Either way, $\alpha_{n}$ is a case in which $C$ obtains even though I fail to know that it does. ${ }^{6}$

Following Williamson, the anti-luminosity argument is often illustrated with the example of feeling cold which, being a phenomenal, introspectable state, might be

\footnotetext{
${ }^{4}$ A near duplicate of a case $\alpha_{x}$ in the sequence will feature a different possible world but will still be centred on me (my counterpart if preferred) and on the same time as $\alpha_{x}$. The two worlds in question will be highly similar overall and identical, at the specified time, with respect to whether $C$ obtains, with respect to the underlying dimension, and with respect to the fact that I'm doing everything that I am in a position to do to determine whether $C$.

${ }^{5}$ Berker $\left(2008\right.$, fn11) argues that a principle like $\mathrm{AL}_{2}$ will lead to the result that there is a possible case in which $\sim C$ clearly obtains, but in which I nevertheless believe that $C$ obtains, even though I am doing everything I am in a position to do to determine whether $C$ obtains. If I believe that $C$ obtains in $\alpha_{n}$ then, by $\mathrm{AL}_{2}$, there is a near duplicate $\beta_{n+1}$ of $\alpha_{n+1}$ in which I believe that $C$ obtains. If $\mathrm{AL}_{2}$ can also be applied to $\beta_{n+1}$, it then follows that there is a near duplicate $\gamma_{n+2}$ of $\beta_{n+2}$ in which I believe that $C$ obtains and so on through the sequence. If $C$ is a good candidate for a luminous condition, then one might think that it is simply not possible for me to believe that $C$ obtains when it clearly does not, in which case we have good reason to reject $\mathrm{AL}_{2}$.

It is important to keep in mind, however, that $\gamma_{n+2}$ need not be a near duplicate of $\alpha_{n+2}$. Even if I believe that $C$ obtains in $\alpha_{n}, \mathrm{AL}_{2}$ is consistent with there being no near duplicate of $\alpha_{n+2}$ in which I believe this. The near duplicate relation is not transitive; the more of these relations that we traverse, the less similar the starting and end points may be. As a result, the case in which I believe that $C$ obtains when it clearly doesn't may count as being extremely dissimilar from any actual case (as Berker acknowledges). I'm inclined to think that this takes the sting out of the objection-the case described should surely be regarded as farfetched, but I see no compelling reason to classify it as impossible. Whatever the truth, though, I won't mount a detailed defence of $\mathrm{AL}_{2}$ here. If this assumption is rejected, then the argument, as stated, will fail of course - not just for $\sim \mathrm{K} \sim \mathrm{KP}$, but for any candidate luminous condition. For further discussion see Srinivasan (2013, pp305-305).

6 The initial formulation of the argument, in terms of 'easy possibility', aligns with the version given by Rosenkranz (2021, Sect. 4.3). When spelled out in terms of near duplicates, the argument is close to the version offered by Srinivasan (2013, Sect. 4) which, for my money, is the most persuasive version currently on offer.
} 
regarded as a paradigm example of a luminous condition. Suppose I wake at 6am feeling bitterly cold, but I gradually warm up throughout the morning until, at noon, I'm feeling uncomfortably hot. Suppose that, throughout this period, I am introspecting as hard as I can, focussing my attention on the question of whether or not I'm feeling cold. The $6 \mathrm{~h}$ from 6 am until noon can be divided up into, say, onemillisecond intervals, giving us our sequence of cases. Over the course of a single millisecond, the change in how I'm feeling will be so slight that I would be in no position to reliably detect it. As a result, if I believe that I'm feeling cold at any point throughout the morning, I could easily believe it one millisecond later. Consider then the final millisecond at which I feel cold. If I don't believe that I'm feeling cold at this point, then I don't know it. If I do believe that I'm feeling cold at this point, then I could easily believe it, falsely, one millisecond later, in which case my belief does not qualify as knowledge. Either way, there is a point at which I'm feeling cold but do not know it.

\section{Rosenkranz on the luminosity of $\sim \mathrm{K} \sim \mathrm{KP}$}

What happens if we try to run the argument for the condition $\sim \mathrm{K} \sim \mathrm{KP}$ (for some proposition $\mathrm{P})$ ? To begin, we need to imagine a sequence that proceeds, via arbitrarily minute increments, from a case in which $\sim \mathrm{K} \sim \mathrm{KP}$ clearly obtains to a case in which $\mathrm{K} \sim \mathrm{KP}$ clearly obtains. Is such a sequence possible? While it is not straightforward to think up a viable example, perhaps the following might work... Suppose I leave my car in a parking lot, and head off to do some shopping. $15 \mathrm{~min}$ later, I have good reason to believe $(\mathrm{P})$ that my car is where I parked it, and I'm in no position to know that I'm in no position to know this $(\sim \mathrm{K} \sim \mathrm{KP})$. Suppose, at this point, I'm standing by the side of a street, when I see a car approaching in the distance. At first, I can't make out any of the details of the car but, as it gradually draws closer, I notice first the colour, then the make, then the bumper stickers, then the license plate... all of which strike me as very familiar. Two minutes later, the car is driving right past me, and it is obvious to me that this is either my car or a near perfect facsimile. At this point, it's clear that I am in a position to know that I'm in no position to know that my car is still where I parked it $(\mathrm{K} \sim \mathrm{KP})$. This two-minute period can be divided up into arbitrarily small intervals-milliseconds, nanoseconds, etc. The shorter the intervals in question, the smaller the change in my visual impression of the car during each. It's very plausible that this narrowing will eventually lead to intervals that are witness to so slight a change that I would be in no position to reliably detect it.

In any case, Rosenkranz is willing to accept the relevant instance of $\mathrm{AL}_{1}$ and grant that we can set up a sequence of the required kind. Suppose, then, that we have our sequence, and suppose that I am, throughout the sequence, using the optimal available method and doing everything that I am in a position to do to determine whether $\sim \mathrm{K} \sim \mathrm{KP}$. Let $\alpha_{n}$ be the last case in which $\sim \mathrm{K} \sim \mathrm{KP}$ and $\alpha_{n+1}$ be the first case in which $\mathrm{K} \sim \mathrm{KP}$. If I believe $\sim \mathrm{K} \sim \mathrm{KP}$ in $\alpha_{n}$, does that mean that I could easily believe $\sim \mathrm{K} \sim \mathrm{KP}$ in $\alpha_{n+1}$ ? According to Rosenkranz, it does not. In $\alpha_{n+1}$, like every other case, I am doing everything that I am in a position to do to determine whether $\sim \mathrm{K} \sim \mathrm{KP}$. By $\mathrm{PK}_{2}$ and $\mathrm{PK}_{3}$, this involves doing everything that $\mathrm{I}$ am in 
a position to do to determine whether $\sim \mathrm{KP}$. But, since $\mathrm{K} \sim \mathrm{KP}$ holds in $\alpha_{n+1}$, this means that I must know $\sim \mathrm{KP}$ in $\alpha_{n+1}$ by $\mathrm{PK}_{1}$. Further, it would be irrational for me to believe $\sim \mathrm{KP}$ and believe $\sim \mathrm{K} \sim \mathrm{KP}$ at the same time and, in order for the former belief to qualify as knowledge, I could not simultaneously hold the latter belief.

Given the way the example is described, and the assumptions $\mathrm{PK}_{1}, \mathrm{PK}_{2}$ and $\mathrm{PK}_{3}$, I could not believe $\sim \mathrm{K} \sim \mathrm{KP}$ in $\alpha_{n+1}$. And the same is clearly true of any near duplicate of $\alpha_{n+1}$. After all, any near duplicate of $\alpha_{n+1}$ will also be a case in which $\mathrm{K} \sim \mathrm{KP}$ and in which I am doing everything that I' $m$ in a position to do to determine whether $\sim \mathrm{K} \sim \mathrm{KP}$. In fact, by Rosenkranz's reasoning, there is no possible case in which these conditions are met and in which I erroneously believe $\sim \mathrm{K} \sim \mathrm{KP}$. Assumption $\mathrm{AL}_{2}$ fails, then, when it comes to the condition $\sim \mathrm{K} \sim \mathrm{KP}$ - even if I believe $\sim \mathrm{K} \sim \mathrm{KP}$ in $\alpha_{n}$, I could not easily (indeed could not possibly, given the set up) believe $\sim \mathrm{K} \sim \mathrm{KP}$ in $\alpha_{n+1}$. Thus, if I believe $\sim \mathrm{K} \sim \mathrm{KP}$ in $\alpha_{n}, \mathrm{AL}_{3}$ presents no obstacle to this belief qualifying as knowledge. ${ }^{7}$

While it's straightforward enough to follow Rosenkranz's reasoning in the abstract, it is somewhat unclear just where it leaves us when it comes to concrete cases. Think again of the above example in which there is a gradual, dawning realisation that an approaching car looks exactly like mine. Are we to think that, at the very nanosecond (indeed the very instant) that $\mathrm{K} \sim \mathrm{KP}$ becomes true, I am perfectly attuned to this change in such a way that I can't possibly make the mistake of believing $\sim \mathrm{K} \sim \mathrm{KP}$ from this point on? Since a nanosecond is too short for me to reliably notice any change in the visual appearances, how am I supposed to manage this? When properly understood, though, Rosenkranz's argument doesn't show (of course) that we have some mysterious power to detect $\mathrm{K} \sim \mathrm{KP}$ from the moment it becomes true. Rather, the set-up of the example ensures that there is a kind of constitutive connection between the obtaining of this condition and our beliefs about it. ${ }^{8}$ Provided that I'm doing everything I am in a position to do to determine

\footnotetext{
7 It's interesting to observe that Rosenkranz's reasoning will apply equally to the condition $\sim \mathrm{KP}$. Suppose we have a sequence running from a case in which $\sim$ KP clearly obtains to a case in which KP clearly obtains and suppose I am doing everything I am in a position to do to determine whether $\sim$ KP obtains. Let $\alpha_{n}$ be the last case in which $\sim \mathrm{KP}$ and $\alpha_{n+1}$ be the first case in which KP. Since, in $\alpha_{n+1}$, I'm doing everything that I'm in a position to do to determine whether $\sim \mathrm{KP}$ it follows, by $\mathrm{PK}_{1}, \mathrm{PK}_{2}$ and $\mathrm{PK}_{3}$, that I know $\mathrm{P}$ in $\alpha_{n+1}$ in which case I don't believe $\sim \mathrm{KP}$ in $\alpha_{n+1}$ (or any near duplicate). If I believe $\sim \mathrm{KP}$ in $\alpha_{n}$, $\mathrm{AL}_{3}$ won't prevent this belief from qualifying as knowledge. By Rosenkranz's reasoning, the anti-luminosity argument, as formulated here, won't work against the condition $\sim \mathrm{KP}$ - and yet, $\sim \mathrm{KP}$ is obviously not a luminous condition, as Rosenkranz himself acknowledges (Rosenkranz, 2021, p58). This should already make us very cautious about concluding that $\sim \mathrm{K} \sim \mathrm{KP}$ may be luminous simply because this version of the anti-luminosity argument fails to show otherwise.

8 Some have attempted to circumvent the anti-luminosity argument by positing constitutive connections between certain conditions and our beliefs about them. Weatherson (2004), Berker (2008) and Ramachandran (2009) all suggest something along the following lines: if one has done everything that one is in a position to do to determine whether one feels cold, then it is necessarily the case that one believes that one feels cold if and only if one does feel cold. It's highly contentious of course whether there really are such constitutive connections between feeling cold and believing that one feels coldbut, if this is accepted, then the argument can be resisted. In a way, the claim that there is a constitutive connection between $\mathrm{K} \sim \mathrm{KP}$ and one failing to believe $\sim \mathrm{K} \sim \mathrm{KP}$, mediated by the fact that I'm doing everything that I' $\mathrm{m}$ in a position to do to determine whether $\sim \mathrm{K} \sim \mathrm{KP}$, is less contentious-it follows from the initial assumptions $\mathrm{PK}_{1}, \mathrm{PK}_{2}$ and $\mathrm{PK}_{3}$ (none of which seems overly controversial) along with a relatively weak condition on knowledge.
} 
whether $\sim \mathrm{K} \sim \mathrm{KP}$, my believing $\sim \mathrm{K} \sim \mathrm{KP}$ would, given $\mathrm{PK}_{1}, \mathrm{PK}_{2}$ and $\mathrm{PK}_{3}$, prevent $\mathrm{K} \sim \mathrm{KP}$ from obtaining.

\section{Adapting the argument}

One possible way to reinstate the anti-luminosity argument would be to strengthen $\mathrm{AL}_{3}$ as follows: if I know that $C$ obtains in a case $\alpha_{x}$, then there is no near duplicate of $\alpha_{x+1}$ in which $C$ is false and in which I invest an at-most-slightly-lower degree of confidence in the proposition that $C$ obtains. This can be motivated by a kind of 'confidence-safety' requirement on knowledge-if one knows a proposition $\mathrm{P}$, then there are no similar cases in which $\mathrm{P}$ is false and, using the same method, one invests an at-most-slightly-lower degree of confidence in $\mathrm{P}^{9}$ (see Williamson, 2000, p101, Berker, 2008, Sect. 4, Srinivasan, 2013, Sect. 5). This may work as a general strategy against those who would seek to undermine the anti-luminosity argument by positing constitutive connections between a given condition and our beliefs about it (Srinivasan, 2013, Sect. 5). In any case, I won't consider this further here. The new variant of the argument that I will offer doesn't involve any modification of $\mathrm{AL}_{3}$ but, rather, a modification of $\mathrm{AL}_{2}$.

As before, let $C$ be a candidate luminous condition, and suppose we can set up a sequence linking a case in which $C$ clearly obtains to a case in which $C$ clearly obtains and in which adjacent cases can be made arbitrarily similar. Suppose that, throughout the sequence, I am using the optimal method and doing everything that I am in a position to do to determine whether $C$ obtains. As before, we accept an appropriate safety condition on knowledge and endorse $\mathrm{AL}_{3}$ : if I know that $C$ obtains in a case $\alpha_{x}$, then I don't falsely believe that $C$ obtains in any near duplicate of $\alpha_{x+1}$.

Instead of $\mathrm{AL}_{2}$, however, we now introduce a new assumption $\mathrm{AL}_{4}$ : if I do not believe that $C$ obtains in a case $\alpha_{x}$, then there is a near duplicate $\beta_{x+1}$ of $\alpha_{x+1}$ and a near duplicate $\beta_{\mathrm{x}-1}$ of $\alpha_{x-1}$ such that I do not believe that $C$ obtains in $\beta_{x+1}$ or $\beta_{x-1}$. $\mathrm{AL}_{4}$ can, I think, be motivated by the same considerations as $\mathrm{AL}_{2}$ - namely, that our powers of discrimination are limited, while the potential similarity of adjacent cases is not. ${ }^{10} \mathrm{AL}_{2}$ implies that, whenever I stop believing that $C$ obtains, I could easily

\footnotetext{
${ }^{9}$ Given the set-up, it's plausible that my confidence in the proposition that $\sim \mathrm{K} \sim \mathrm{KP}$ will gradually decrease as the sequence progresses. By Rosenkranz's reasoning, I cannot believe $\sim \mathrm{K} \sim \mathrm{KP}$ in $\alpha_{n+1}$. And yet, if $\alpha_{n+1}$ is the first case in which I cease to believe $\sim \mathrm{K} \sim \mathrm{KP}$ it's plausible that my confidence in this proposition will be just below the threshold needed for outright belief (or, at the very least, that there is a near duplicate of $\alpha_{n+1}$ in which my confidence in this proposition will be just below the threshold needed for outright belief). Since it's also plausible that my confidence in $\sim \mathrm{K} \sim \mathrm{KP}$ in $\alpha_{n}$ would be only just above the threshold for outright belief, by the confidence-safety version of $\mathrm{AL}_{3}$, this belief would not qualify as knowledge. I won't explore this version of the anti-luminosity argument here. For what it's worth, I'm inclined to think that the 'confidence-safety' condition is problematic, and the most persuasive versions of the anti-luminosity argument are those that steer clear of this commitment.

${ }^{10} \mathrm{AL}_{4}$ could still be accepted by someone who is persuaded by Berker's argument against $\mathrm{AL}_{2}$ (discussed in footnote 5). $\mathrm{AL}_{4}$ will imply, by a kind of mirror image of Berker's reasoning, that there is a possible (but potentially highly dissimilar) case in which $C$ clearly obtains but I fail to believe that it does. This may be significantly easier to accept than the existence of a possible case in which I believe that $C$ obtains even though it clearly does not. The former case, unlike the latter, need involve no error or misjudgement on my part.
} 
have stopped a moment later, while $\mathrm{AL}_{4}$ implies that, whenever I stop believing that $C$ obtains, I could easily have stopped a moment earlier. Both thoughts are plausible for the same reason-the changes from moment to moment are too slight for my beliefs to be perfectly attuned to them.

We now have the tools needed to prove that $C$ cannot be a luminous condition. Let $\alpha_{n}$ be the last case in the sequence in which $C$ obtains and $\alpha_{n+1}$ be the first case in which $\sim C$ obtains. If I believe that $C$ obtains in $\alpha_{n+1}$, then, by $\mathrm{AL}_{3}$, I don't know that $C$ obtains in $\alpha_{n}$. If I don't believe that $C$ obtains in $\alpha_{n+1}$ then, by $\mathrm{AL}_{4}$, there is a near duplicate $\beta_{n}$ of $\alpha_{n}$ in which I don't believe that $C$ obtains and, thus, don't know it. Either way, we have a case $\left(\alpha_{n}\right.$ or $\left.\beta_{n}\right)$ in which $C$ obtains, even though I fail to know that it does.

Rosenkranz's argument against the relevant instance of $\mathrm{AL}_{2}$ hinged on the fact that, as long I am doing everything that I am in a position to do to determine whether $\sim \mathrm{K} \sim \mathrm{KP}, \mathrm{K} \sim \mathrm{KP}$ will be incompatible with my believing $\sim \mathrm{K} \sim \mathrm{KP}$. No such argument can be mounted against the relevant instance of $\mathrm{AL}_{4}$ - at least, not on the basis of the assumptions $\mathrm{PK}_{1}, \mathrm{PK}_{2}$ and $\mathrm{PK}_{3}$. Perhaps we could motivate other assumptions about being in a position to know that would serve this purpose, but these three, relatively minimal, assumptions are not enough-they are perfectly compatible with $\sim \mathrm{K} \sim \mathrm{KP}$ being true, and with my failing to believe $\sim \mathrm{K} \sim \mathrm{KP}$, even if I am doing everything that $\mathrm{I}$ am in a position to do to determine whether $\sim \mathrm{K} \sim \mathrm{KP}$.

As noted above, Rosenkranz doesn't mean to ascribe us any mysterious power to perfectly detect the condition $\sim \mathrm{K} \sim \mathrm{KP}-\mathrm{it}$ is the constitutive connections between $\sim \mathrm{K} \sim \mathrm{KP}$ and our beliefs about it that enable us to answer the original antiluminosity argument. But for $\sim \mathrm{K} \sim \mathrm{KP}$ to be a genuinely luminous condition may, after all, require a mysterious power on our part. Constitutive connections may ensure that my belief in $\sim \mathrm{K} \sim \mathrm{KP}$ cannot outrun its truth as we progress through the sequence. If I am doing everything I am in a position to do to determine whether $\sim \mathrm{K} \sim \mathrm{KP}$, then I cannot make a type II error and believe that $\sim \mathrm{K} \sim \mathrm{KP}$ obtains when it doesn't-for the belief is, in a way, self-fulfilling. But it is less clear that there are any constitutive connections which will guarantee that the truth of $\sim \mathrm{K} \sim \mathrm{KP}$ cannot outrun my belief. Even if I am doing everything I am in a position to do to determine whether $\sim \mathrm{K} \sim \mathrm{KP}$, it is less clear whether there are any constitutive connections guarding against the possibility of a type $I$ error. $^{11}$

\footnotetext{
11 Some of Rosenkranz's reasoning from 4.2 might be deployed to try and show that I could not fail to believe $\sim \mathrm{K} \sim \mathrm{KP}$ in a case in which $\sim \mathrm{K} \sim \mathrm{KP}$ holds, and in which $\mathrm{I}$ am doing everything that $\mathrm{I}$ am in a position to do to determine whether $\sim \mathrm{K} \sim \mathrm{KP}$. Consider a case in which $\mathrm{I}$ have done everything that $\mathrm{I}$ am in a position to do to determine whether $\sim \mathrm{K} \sim \mathrm{KP}$, but I do not believe $\sim \mathrm{K} \sim \mathrm{KP}$. What is my attitude to the embedded proposition $\sim \mathrm{KP}$ ? If I've done everything that $\mathrm{I}$ am in a position to do to determine whether $\sim \mathrm{K} \sim \mathrm{KP}$, then, by $\mathrm{PK}_{2}$ and $\mathrm{PK}_{3}$, I've done everything that $\mathrm{I}$ am in a position to do determine whether $\sim \mathrm{KP}$. Thus, if I do believe $\sim \mathrm{KP}$, it looks as though this belief will count as safe, by the reasoning in $\mathrm{n} 7$. If I have done everything that I am in a position to do to determine whether a proposition holds and have arrived at a safe belief in that proposition, then one might argue that my belief should count as knowledge (this is something like Rosenkranz's (6) - pp54-55). It would then follow that $\mathrm{K} \sim \mathrm{KP}$ must hold in this case. What if I don't believe $\sim \mathrm{KP}$ ? One might argue that I should then be in a position to know that I don't believe it and, thus, to know that I don't know it, giving us K k KP. If we assume that
} 
Rosenkranz's attempt to save $\sim \mathrm{K} \sim \mathrm{KP}$ from the anti-luminosity argument may, in the end, be unsuccessful. But there is, nevertheless, much that we learn by carefully thinking this through. Most obviously, we learn something about the anti-luminosity argument itself and the kinds of assumptions that can be used to drive it. And perhaps we learn something significant about knowledge and justification as well. When it comes to $\sim \mathrm{K} \sim \mathrm{KP}$ - which is equivalent to JP on Rosenkranz's theory-it may be that a certain kind of mistake really is impossible. If one is using the optimal method to determine whether this condition holds, then it cannot deliver a false positive.

Acknowledgements This paper was presented at a symposium on Justification as Ignorance held in July 2021. Thanks to all of those who participated on this occasion. Thanks also to Sven Rosenkranz and to an anonymous referee for helpful written comments on earlier drafts of this material.

Author contribution Not applicable.

Data availability My manuscript has no associated data.

Code availability Not applicable.

\section{Declarations}

Conflict of interest The author declares no competing interests.

Open Access This article is licensed under a Creative Commons Attribution 4.0 International License, which permits use, sharing, adaptation, distribution and reproduction in any medium or format, as long as you give appropriate credit to the original author(s) and the source, provide a link to the Creative Commons licence, and indicate if changes were made. The images or other third party material in this article are included in the article's Creative Commons licence, unless indicated otherwise in a credit line to the material. If material is not included in the article's Creative Commons licence and your intended use is not permitted by statutory regulation or exceeds the permitted use, you will need to obtain permission directly from the copyright holder. To view a copy of this licence, visit http://creativecommons.org/licen ses/by/4.0/.

\section{References}

Berker, S. (2008). 'Luminosity regained.' Philosophers' Imprint, v8(2), 1-22.

Pryor, J. (2001). 'Highlights of recent epistemology.' British Journal for the Philosophy of Science, v52(1), 95-124.

Ramachandran, M. (2009). 'Anti-luminosity: Four unsuccessful strategies.' Australasian Journal of Philosophy, v87(4), 659-673.

Footnote 11 (continued)

I must also be in a position to know that I have done everything that I'm in a position to do to determine whether $\sim \mathrm{KP}$, then, from the fact that I don't know it, I could conclude that I'm in no position to know it, giving us $\mathrm{K} \sim \mathrm{K} \sim \mathrm{KP}$. But if I'm in a position to know $\sim \mathrm{K} \sim \mathrm{KP}$ and I've done everything that I'm in a position to do to determine whether $\sim \mathrm{K} \sim \mathrm{KP}$, then I must know, and believe, $\sim \mathrm{K} \sim \mathrm{KP}$ contrary to stipulation. If I have done everything that $\mathrm{I}$ am in a position to do to determine whether $\sim \mathrm{K} \sim \mathrm{KP}$, but $\mathrm{I}$ do not believe $\sim \mathrm{K} \sim \mathrm{KP}$, then the only possibility, it seems, is that $\mathrm{K} \sim \mathrm{KP}$ holds. Whatever we ultimately make of this reasoning, though, it's important to emphasise that it relies on assumptions that are far more substantial, and far more controversial, than $\mathrm{PK}_{1}, \mathrm{PK}_{2}$ and $\mathrm{PK}_{3}$. 
Rosenkranz, S. (2021). Justification as Ignorance. Oxford University Press.

Srinivasan, A. (2013). 'Are we luminous?' Philosophy and Phenomenological Research, v90(2), 294-319.

Weatherson, B. (2004). 'Luminous margins.' Australasian Journal of Philosophy, v82(3), 373-383.

Williamson, T. (2000). Knowledge and its Limits. Oxford University Press. 